\title{
Strong ellipticity and progressive waves in elastic materials with voids
}

\author{
By Stan Chiriţă $\breve{A}^{1} \&$ IONEL-Dumitrel GhiBA ${ }^{2}$ \\ ${ }^{1}$ Faculty of Mathematics, Al. I. Cuza University of Iaşi, Blvd. Carol I, \\ no. 11, 700506 - Iaşi, Romania \\ 2 "Octav Mayer" Mathematics Institute, Romanian Academy of Science, \\ Iaşi Branch, Blvd. Carol I, no. 8, 700506 - Iaşi, Romania
}

In the present paper we investigate a model for propagating progressive waves associated with the voids within the framework of a linear theory of porous media. Due to the use of lighter materials in modern buildings and noise concerns in the environment such models for progressive waves are of much interest to the building industry. Analysis of such waves is also of interest in acoustic microscopy where the identification of material defects is of paramount importance to industry and medicine. Our analysis is based on the strong ellipticity of the poroelastic materials. We illustrate the model of progressive wave propagation for isotropic and transversely isotropic porous materials. We also study the propagation of harmonic plane waves in porous materials including the thermal effect.

Keywords: Strong ellipticity; Progressive waves; Elastic materials with voids; Thermoelastic materials with voids

2000 Mathematics Subject Classification: 74E20, 74J05, 74F05.

\section{Introduction}

Wave motion in an elastic material containing voids is an area with immense potential for practical applications. There are developed many theories which describe the behaviour of materials with voids and which proved to be useful in practice. In seismic zones buildings are constructed with much lighter porous materials and typically have thinner walls. As a consequence, there is a great need to study the acoustic properties of porous materials including the nature of the solid elastic matrix and the gas filling the pores, and the influence of temperature on these quantities. But seismology represents only one of the many fields where the theory of materials with voids is applied. Medicine, various branches of biology and oil exploration industry are other important fields of application.

In this paper we consider the theory of materials with voids introduced by Cowin \& Nunziato (1983). The theory of elastic materials with voids is the simplest extension of the classical theory of elasticity. The basic idea of this theory is to suppose that there is a distribution of voids throughout the elastic body. Consequently, the bulk density is written as the product of two fields: the matrix material density field and the volume fraction field. This representation introduces an additional degree of kinematic freedom and it was employed previously by Goodman \& Cowin (1972) to develop a continuum theory of granular materials. The first investigations in the theory of thermoelastic materials with voids are due to Nunziato \& Cowin (1979) and Ieşan (1986). The Nunziato-Cowin theory has enjoyed much success in predicting various effects especially within the remit of linear theory, see e.g. Ciarletta \& Ieşan (1993), Ieşan (2004) and the recent work of Quintanilla (2001), Casas \& Quintanilla (2005), Magaña \& Quintanilla (2006, 2007), Ghiba (2008a), and the references therein. The intended applications of the theory concern the manufacture of building materials, e.g. plasterboard, brick, concrete. 
The first aim of the present study is to establish necessary and sufficient conditions characterizing the strong ellipticity of materials with voids. Such conditions are important in discussing uniqueness, wave propagation (see e.g. Gurtin (1972), p. 86), loss of ellipticity in the context of the nonlinear elasticity of fibre-reinforced materials (Merodio \& Ogden 2003) and also in the study of spatial behaviour of the constrained anisotropic cylinders (Chiriţă \& Ciarletta 2006) or of constrained rectangular plates (Ghiba 2008b).

Simpson and Spector (1983) treat the strong ellipticity condition for isotropic nonlinearly elastic materials. They establish necessary and sufficient conditions for the strong ellipticity of the equations governing an isotropic (compressible) nonlinearly elastic material at equilibrium. The main tool in their analysis consists in a representation theorem for copositive matrices. A review on strong ellipticity for isotropic nonlinearly elastic materials is given by Dacorogna (2001).

Notable contribution concerning the strong ellipticity for the class of transverse isotropic linearly elastic materials are the work by Payton (1983) and the recent results by Padovani (2002), Merodio \& Ogden (2003) and Chiriţă (2006). For other symmetries of elastic materials the strong ellipticity is studied by Chiriţă et al. (2007), Chiriţă \& Danescu (2008) and Han et al. (2009).

In this paper, general necessary and sufficient conditions are established for anisotropic centro-symmetric materials with voids but two types of materials are discussed in detail: isotropic and transversely isotropic elastic materials with voids. The theory of isotropic materials is a good approach for many problems in engineering and biological sciences. Ding et al. (2006) have presented methods for studying different types of problems which arise in the theory of transversely isotropic elastic materials. Besides the well known applications of this type of material in the mechanics of rocks (Wyllie 1999; Jaeger 2007), transversely isotropic materials are very useful in many branches of biology (Humphrey 2002; Dong \& Guo, 2004; Goldmann et al. 2005). The recent studies of fiber-reinforced composites (Spencer 1992; Bunsell \& Renard, 2005) and the modern technologies also encourage the study of transversely isotropic materials.

In the second part of this paper we study the propagation of progressive waves in elastic materials with voids. We also consider the propagation of waves in thermoelastic isotropic materials with voids. We outline that the problem of wave propagation in materials with voids was studied in various works (Nunziato \& Walsh 1977, 1978; Nunziato et al. 1978; Cowin \& Nunziato 1983; Ciarletta \& Straughan 2007a,b; Ciarletta et al. 2007) and a comprehensive review of the results can be found in the book by Straughan (2008). The use of acceleration waves and related analysis have proved extremely useful in recent investigations of wave motion in various dispersive and random media, in a variety of thermodynamic states, see e.g. Ostoja-Starzewski \& Trebicki (1999), Puri \& Jordan (2004), Quintanilla \& Straughan (2004), Christov \& Jordan (2005), Jordan \& Christov (2005); Jordan \& Puri (2005), Christov et al. (2006).

The present mathematical results can be used in laboratories for studying the specific properties of materials with voids.

\section{Basic equations of elastic model with voids}

Throughout this section $B$ is a bounded regular region of three-dimensional Euclidean space. We let $\bar{B}$ denote the closure of $B$, call $\partial B$ the boundary of $B$, and designate by $\mathbf{n}$ the outward unit normal of $\partial B$. We assume that the body occupying $B$ is a linearly elastic solid with voids. The body is referred to a fixed system of rectangular Cartesian axes $O x_{i}(i=1,2,3)$. Throughout this paper Latin indices have the range 1, 2, 3, Greek indices have the range 1, 2 and the usual summation convention is employed. Moreover, subscripts preceded by a comma denote for partial differentiation with respect to the corresponding coordinate, while a superposed dot means for the time differentiation.

Let $\mathbf{u}$ be the displacement vector field over $B$, and let $\varphi$ be the volume fraction field 
over $B$. Then the components $e_{r s}$ of the linear strain tensor are given by

$$
e_{r s}=\frac{1}{2}\left(u_{r, s}+u_{s, r}\right)
$$

and the constitutive equations for a homogeneous and anisotropic elastic solid with voids are (cf. Cowin \& Nunziato (1983))

$$
\begin{aligned}
t_{r s} & =C_{r s m n} e_{m n}+B_{r s} \varphi+D_{r s k} \varphi_{, k}, \\
h_{r} & =A_{r s} \varphi_{, s}+D_{m n r} e_{m n}+d_{r} \varphi, \\
g & =-B_{r s} e_{r s}-\xi \varphi-d_{r} \varphi_{, r} .
\end{aligned}
$$

Here we have used the notations: $t_{r s}$ are the components of the stress tensor, $h_{r}$ are the components of the equilibrated stress vector, $g$ is the intrinsic equilibrated body force and $A_{r s}, B_{r s}, C_{r s m n}, D_{r s k}, d_{r}$ and $\xi$ are constant constitutive coefficients satisfying the following symmetries

$$
\begin{aligned}
A_{r s} & =A_{s r}, \quad B_{r s}=B_{s r}, \quad D_{r s k}=D_{s r k}, \\
C_{m n r s} & =C_{r s m n}=C_{n m r s} .
\end{aligned}
$$

The specific internal energy $W$ is given by

$$
\begin{aligned}
W(\mathbf{e}, \varphi, \operatorname{grad} \varphi) & =\frac{1}{2} C_{m n r s} e_{m n} e_{r s}+\frac{1}{2} \xi \varphi^{2}+\frac{1}{2} A_{r s} \varphi_{, r} \varphi_{, s}+ \\
& +B_{r s} e_{r s} \varphi+D_{r s k} e_{r s} \varphi_{, k}+d_{r} \varphi \varphi_{, r}
\end{aligned}
$$

The specific internal energy $W_{0}$ for an isotropic and homogeneous elastic material with voids is defined by

$$
W_{0}(\mathbf{e}, \varphi, \operatorname{grad} \varphi)=\mu e_{r s} e_{r s}+\frac{1}{2} \lambda e_{r r} e_{s s}+\frac{1}{2} \xi \varphi^{2}+\frac{1}{2} \alpha \varphi_{, r} \varphi_{, r}+\beta \varphi e_{r r},
$$

where $\lambda, \mu, \alpha, \beta$ and $\xi$ are constant constitutive coefficients.

The transverse isotropy is characterized by the symmetry group consisting of unit tensor 1 and the rotations $\mathbb{R}_{\mathbf{e}_{3}}^{\theta}(0<\theta<2 \pi)$, that is the orthogonal tensor corresponding to a right-handed rotation through the angle $\theta, 0<\theta<2 \pi$, about the axis in the direction of the unit vector $\mathbf{e}_{3}$ ). For a transversely isotropic elastic material with voids the specific internal energy $W_{1}$ is defined by

$$
\begin{gathered}
W_{1}(\mathbf{e}, \varphi, \operatorname{grad} \varphi)=\frac{1}{2} c_{11}\left(e_{11}^{2}+e_{22}^{2}\right)+\frac{1}{2} c_{33} e_{33}^{2}+2 c_{55}\left(e_{13}^{2}+e_{23}^{2}\right)+2 c_{66} e_{12}^{2}+ \\
+\frac{1}{2} \xi \varphi^{2}+\frac{1}{2} a_{11}\left(\varphi_{, 1}^{2}+\varphi_{, 2}^{2}\right)+\frac{1}{2} a_{33} \varphi_{, 3}^{2}+c_{12} e_{11} e_{22}+c_{13}\left(e_{11}+e_{22}\right) e_{33}+ \\
+b_{11}\left(e_{11}+e_{22}\right) \varphi+b_{33} e_{33} \varphi
\end{gathered}
$$

where

$$
\begin{gathered}
c_{11}=C_{1111}, \quad c_{33}=C_{3333}, \quad c_{55}=C_{1313}, \quad c_{13}=C_{1133}, \quad c_{12}=C_{1122}, \\
c_{66}=\frac{1}{2}\left(c_{11}-c_{12}\right), \quad a_{11}=A_{11}, \quad a_{33}=A_{33}, \quad b_{11}=B_{11}, \quad b_{33}=B_{33} .
\end{gathered}
$$

The other constitutive coefficients are zero.

In the absence of the body force and the extrinsic equilibrated body force, the equations of motion of the elastic material with voids are given by (cf. Cowin \& Nunziato $(1983))$

$$
\begin{aligned}
t_{r s, r} & =\varrho \ddot{u}_{s}, \\
h_{r, r}+g & =\varrho \varkappa \ddot{\varphi} .
\end{aligned}
$$


If we substitute (2.1) and (2.2) into (2.8) we obtain the basic equations in terms of the displacement and volume fraction fields in the form

$$
\begin{aligned}
\left(C_{m n r s} u_{r, s}+B_{m n} \varphi+D_{m n k} \varphi_{, k}\right)_{, m} & =\varrho \ddot{u}_{n}, \\
\left(A_{m n} \varphi_{, n}+D_{r s m} u_{r, s}+d_{m} \varphi\right)_{, m}-B_{r s} u_{r, s}-\xi \varphi-d_{r} \varphi_{, r} & =\varrho \varkappa \ddot{\varphi},
\end{aligned}
$$

which for isotropic materials with voids becomes

$$
\begin{aligned}
\mu \Delta u_{r}+(\lambda+\mu) u_{s, s r}+\beta \varphi_{, r} & =\varrho \ddot{u}_{r}, \\
\alpha \Delta \varphi-\xi \varphi-\beta u_{r, r} & =\varrho \varkappa \ddot{\varphi}
\end{aligned}
$$

where $\Delta$ is the Laplace operator.

We have to note that the specific internal energy $W_{0}$ is a positive definite quadratic form in terms of the variables $\left(e_{11}, e_{22}, e_{33}, e_{12}, e_{23}, e_{31}, \varphi, \varphi, 1, \varphi, 2, \varphi_{, 3}\right)$ if and only if

$$
\mu>0, \quad \alpha>0, \quad \xi>0, \quad\left(\lambda+\frac{2}{3} \mu\right) \xi>\beta^{2},
$$

while $W_{1}$ is a positive definite quadratic form in terms of the variables $\left(e_{11}, e_{22}, e_{33}\right.$, $\left.e_{12}, e_{23}, e_{31}, \varphi, \varphi, 1, \varphi, 2, \varphi, 3\right)$ if and only if

$$
\begin{gathered}
c_{11}>0, \quad c_{33}>0, \quad c_{55}>0, \quad \xi>0, \quad a_{11}>0, \quad a_{33}>0, \\
\left|c_{12}\right|<c_{11}, \quad\left|c_{13}\right|<\sqrt{\frac{1}{2}\left(c_{11}+c_{12}\right) c_{33},} \\
\left(c_{13}-\frac{b_{11} b_{33}}{\xi}\right)^{2}<\left(c_{33}-\frac{b_{33}^{2}}{\xi}\right)\left[\frac{1}{2}\left(c_{11}+c_{12}\right)-\frac{b_{11}^{2}}{\xi}\right] .
\end{gathered}
$$

\section{Strongly elliptic elastic materials with voids}

We will say that the elastic material with voids is strongly elliptic if

$$
W(\mathbf{A}, \eta, \boldsymbol{\zeta})>0, \quad \text { for all }(\mathbf{A}, \eta, \boldsymbol{\zeta}) \neq \mathbf{0},
$$

with $\mathbf{A}=\mathbf{m} \otimes \mathbf{n}, \mathbf{m}, \mathbf{n}$ and $\boldsymbol{\zeta}$ arbitrary vectors and $\eta$ an arbitrary scalar. The strongly elliptic condition (3.1) can be written in an explicit way in the following form

$$
\begin{gathered}
\frac{1}{2} C_{m n r s} m_{m} m_{r} n_{n} n_{s}+\frac{1}{2} \xi \eta^{2}+\frac{1}{2} A_{r s} \zeta_{r} \zeta_{s}+ \\
+B_{r s} m_{r} n_{s} \eta+D_{r s k} m_{r} n_{s} \zeta_{k}+d_{r} \eta \zeta_{r}>0, \quad \text { for all }(\mathbf{m} \otimes \mathbf{n}, \eta, \boldsymbol{\zeta}) \neq \mathbf{0},
\end{gathered}
$$

where $\mathbf{m}=\left(m_{1}, m_{2}, m_{3}\right), \mathbf{n}=\left(n_{1}, n_{2}, n_{3}\right)$ and $\boldsymbol{\zeta}=\left(\zeta_{1}, \zeta_{2}, \zeta_{3}\right)$.

We can observe that the strong ellipticity condition (3.1) assures the strong ellipticity of the system of partial differential equations (2.9) (Fichera 1965). We have to note that the strong ellipticity condition (3.2) implies that

$$
\begin{gathered}
C_{m n r s} m_{m} m_{r} n_{n} n_{s}>0, \quad \text { for all unit vectors } \mathbf{n}, \mathbf{m}, \\
\quad \xi>0, \\
A_{r s} \zeta_{r} \zeta_{s}>0, \quad \text { for all unit vectors } \zeta .
\end{gathered}
$$

The inequality (3.2) is the general strong ellipticity condition for an arbitrary anisotropic poroelastic body. Our objective here consists of finding explicit necessary and sufficient conditions characterising the strong ellipticity of elastic materials with voids. However, we believe the results one obtains when the porous body has a centre of 
symmetry are revealing. When the poroelastic body possesses a centre of symmetry, the terms $D_{r s k}$ and $d_{m}$ are zero. Thus, for the centro-symmetric elastic materials with voids the strong ellipticity condition $(3.2)$ reduce to

$$
\frac{1}{2} C_{m n r s} m_{m} m_{r} n_{n} n_{s}+\frac{1}{2} \xi \eta^{2}+\frac{1}{2} A_{r s} \zeta_{r} \zeta_{s}+B_{r s} m_{r} n_{s} \eta>0, \quad \text { for all }(\mathbf{m} \otimes \mathbf{n}, \eta, \boldsymbol{\zeta}) \neq \mathbf{0}
$$

This condition is equivalent to the following inequalities

$$
\begin{array}{r}
A_{r s} \zeta_{r} \zeta_{s}>0 \text { for all unit vectors } \boldsymbol{\zeta} \\
\xi \eta^{2}+2 B_{r s} m_{r} n_{s} \eta+C_{m n r s} m_{m} m_{r} n_{n} n_{s}>0
\end{array}
$$

for all real scalar $\eta$ and unit vectors $\mathbf{m}, \mathbf{n}$.

Further, regarding (3.8) as a quadratic in $\eta \in \mathbb{R}$, we deduce that it is equivalent to the following inequalities

$$
\xi>0
$$

$$
\left(B_{r s} m_{r} n_{s}\right)^{2}<\xi C_{m n r s} m_{m} m_{r} n_{n} n_{s} \text { for all unit vectors } \mathbf{m}, \mathbf{n} \text {. }
$$

This last condition can be written as

$$
\left(\xi C_{m n r s}-B_{m n} B_{r s}\right) m_{m} m_{r} n_{n} n_{s}>0 \text { for all unit vectors } \mathbf{m}, \mathbf{n},
$$

that is the tensor

$$
\mathcal{C}_{m n r s}=\xi C_{m n r s}-B_{m n} B_{r s}
$$

is strongly elliptic.

Concluding, we deduce that a centro-symmetric elastic material with voids is strongly elliptic if and only if $A_{r s}$ is a positive definite tensor, $\xi>0$ and $\mathcal{C}_{m n r s}$ is strongly elliptic tensor.

In what follows we will establish explicit necessary and sufficient conditions characterizing the strong ellipticity of the isotropic and transversely isotropic elastic materials with voids.

\section{(a) Isotropic elastic materials with voids}

In this subsection we characterize the strong ellipticity for the class of isotropic elastic materials with voids. To this end we note that the strong ellipticity condition (3.6) becomes

$$
\mu m_{r} m_{r} n_{s} n_{s}+\frac{1}{2} \lambda m_{r} n_{r} m_{s} n_{s}+\beta \eta m_{r} n_{r}+\frac{1}{2} \xi \eta^{2}+\frac{1}{2} \alpha \zeta_{r} \zeta_{r}>0
$$

and it is equivalent to

$$
\alpha>0, \quad \xi>0
$$

and

$$
\beta^{2} m_{r} n_{r} m_{s} n_{s}<\xi\left[2 \mu m_{r} m_{r} n_{s} n_{s}+\lambda m_{r} n_{r} m_{s} n_{s}\right] \text {, for all unit vectors } \mathbf{m}, \mathbf{n} \text {. }
$$

Further, we recall that we can write $(3.15)$ in the form

$$
\begin{gathered}
2 \xi \mu\left[\left(m_{1} n_{2}-m_{2} n_{1}\right)^{2}+\left(m_{2} n_{3}-m_{3} n_{2}\right)^{2}+\left(m_{3} n_{1}-m_{1} n_{3}\right)^{2}\right]+ \\
+\left[\xi(\lambda+2 \mu)-\beta^{2}\right]\left(m_{1} n_{1}+m_{2} n_{2}+m_{3} n_{3}\right)^{2}>0
\end{gathered}
$$


and note that it is equivalent to the following inequalities

$$
\begin{aligned}
\xi \mu & >0, \\
\xi(\lambda+2 \mu)-\beta^{2} & >0 .
\end{aligned}
$$

Concluding, we can say that an isotropic elastic material with voids is strongly elliptic if and only if the elastic coefficients satisfy the following inequalities

$$
\begin{aligned}
\mu & >0, \\
\xi & >0, \\
\alpha & >0, \\
\xi(\lambda+2 \mu) & >\beta^{2} .
\end{aligned}
$$

It appears clear from the relations (2.11) and (3.18) that the positive definiteness assumption of the specific internal energy $W_{0}$ implies the strong ellipticity of the elastic material with voids.

\section{(b) Transversely isotropic elastic materials with voids}

Many natural and man-made materials are classified as transversely isotropic. Such materials are characterized by the fact that one can find a line, that allows a rotation of the material about it without changing its elastic properties. The plane, which is perpendicular to this line (the axis of rotational symmetry) is called a plane of elastic symmetry or plane of isotropy. Modern examples for such materials are laminates made of randomly oriented chopped fibers that are in general placed in a certain plane. The effective elastic material properties for a bundled structure have no preferred direction in that plane, which then becomes a plane of elastic symmetry. Hence, each plane that contains the axis of rotation is a plane of symmetry, and therefore, transversely isotropic elastic material admits an infinite number of elastic symmetries. Thus, the transverse isotropy is appropriate to real materials having a laminate or a bundled structure.

The strong ellipticity condition (3.6) reduces to

$$
\begin{gathered}
c_{66}\left(n_{1} m_{2}-n_{2} m_{1}\right)^{2}+c_{11}\left(n_{1} m_{1}+n_{2} m_{2}\right)^{2}+c_{33} n_{3}^{2} m_{3}^{2}+c_{55}\left(n_{3}^{2} m_{1}^{2}+n_{1}^{2} m_{3}^{2}+\right. \\
\left.+n_{3}^{2} m_{2}^{2}+n_{2}^{2} m_{3}^{2}\right)+\xi \eta^{2}+a_{11}\left(\zeta_{1}^{2}+\zeta_{2}^{2}\right)+a_{33} \zeta_{3}^{2}+2\left(c_{13}+c_{55}\right)\left(n_{1} m_{1}+\right. \\
\left.+n_{2} m_{2}\right) n_{3} m_{3}+2 b_{11}\left(n_{1} m_{1}+n_{2} m_{2}\right) \eta+2 b_{33} n_{3} m_{3} \eta>0,
\end{gathered}
$$

and it is equivalent to the inequalities

$$
a_{11}>0, \quad a_{33}>0, \quad \xi>0
$$

and the strong ellipticity of the elasticity tensor given by

$$
\left(\begin{array}{cccccc}
\xi c_{11}-b_{11}^{2} & \xi c_{12}-b_{11}^{2} & \xi c_{13}-b_{11} b_{33} & 0 & 0 & 0 \\
\xi c_{12}-b_{11}^{2} & \xi c_{11}-b_{11}^{2} & \xi c_{13}-b_{11} b_{33} & 0 & 0 & 0 \\
\xi c_{13}-b_{11} b_{33} & \xi c_{13}-b_{11} b_{33} & \xi c_{33}-b_{33}^{2} & 0 & 0 & 0 \\
0 & 0 & 0 & \xi c_{55} & 0 & 0 \\
0 & 0 & 0 & 0 & \xi c_{55} & 0 \\
0 & 0 & 0 & 0 & 0 & \xi c_{66}
\end{array}\right)
$$

In view of the results established by Merodio and Ogden (2003), Chiriţă (2006) and Chiriţă et al. (2007) the elasticity tensor defined in (3.21) is strongly elliptic if and only if

$$
\xi c_{11}-b_{11}^{2}>0, \quad \xi c_{33}-b_{33}^{2}>0
$$




$$
\begin{gathered}
\xi c_{55}>0, \quad \xi\left(c_{11}-c_{12}\right)>0 \\
\left|c_{13}+c_{55}-\frac{b_{11} b_{33}}{\xi}\right|<c_{55}+\sqrt{\left(c_{11}-\frac{b_{11}^{2}}{\xi}\right)\left(c_{33}-\frac{b_{33}^{2}}{\xi}\right)} .
\end{gathered}
$$

Concluding, we deduce that a transversely isotropic elastic material with voids is strongly elliptic if and only if the constitutive coefficients satisfy the relations (3.20), (3.22), (3.23) and (3.24).

\section{Progressive waves in centro-symmetric elastic materials with voids}

Throughout this section we will consider plane progressive waves in elastic materials with voids having a centre of symmetry, that is materials for which we have

$$
D_{i j k}=0, \quad d_{r}=0 .
$$

By a plane progressive wave in a centro-symmetric elastic material with voids we mean a couple of functions $\left(u_{r}, \varphi\right)$ on $\mathbb{R}^{3} \times(-\infty, \infty)$ of the following form

$$
\begin{aligned}
u_{r}(\mathbf{x}, t) & =U_{r} \Lambda\left(n_{s} x_{s}-c t\right), \\
\varphi(\mathbf{x}, t) & =\Phi \Gamma\left(n_{s} x_{s}-c t\right),
\end{aligned}
$$

where:

(i) $\Lambda$ and $\Gamma$ are real-valued functions of class $C^{2}$ on $(-\infty, \infty)$ with

$$
\frac{d^{2} \Gamma}{d s^{2}}=-\Gamma \neq 0, \quad \frac{d \Lambda}{d s}=\Gamma
$$

(ii) $\mathbf{U}=\left(U_{1}, U_{2}, U_{3}\right)$ is the displacement amplitude vector, $\mathbf{n}=\left(n_{1}, n_{2}, n_{3}\right)$ is a unit vector which represents the direction of propagation and $\left(U_{1}, U_{2}, U_{3}, \Phi\right) \neq \mathbf{0}$;

(iii) $c$ is the speed of propagation.

We say that the progressive wave is longitudinal if $\mathbf{U}$ and $\mathbf{n}$ are linearly dependent, that is $\mathbf{U} \times \mathbf{n}=\mathbf{0}$. It is transverse when $\mathbf{U}$ and $\mathbf{n}$ are perpendicular, that is $\mathbf{U} \cdot \mathbf{n}=0$. For any given constant $\gamma$ and for any given time $t$ the displacement and volume fraction fields (4.2) are constants on the plane $n_{s} x_{s}-c t=\gamma$. This last plane is perpendicular to $\mathbf{n}$ and it is moving with the speed $c$ in the direction $\mathbf{n}$.

These plane waves are important in that they furnish valuable information concerning the propagation characteristics of elastic materials with voids.

If we substitute the plane progressive wave described by (4.2) into basic equations (2.9) and then we take into consideration the assumptions described into (4.1) and (4.3), we see that such waves are possible in an elastic material with voids if and only if

$$
\begin{aligned}
\left(C_{m n r s} n_{s} n_{m}-\varrho c^{2} \delta_{r n}\right) U_{r}+B_{m n} n_{m} \Phi & =0 \\
B_{r s} n_{s} U_{r}+\left(\xi+A_{r s} n_{r} n_{s}-\varrho \varkappa c^{2}\right) \Phi & =0 .
\end{aligned}
$$

Such linear algebraic system admits a non-zero solution $\left(U_{1}, U_{2}, U_{3}, \Phi\right)$ if and only if $c^{2}$ satisfies the following algebraic equation

$$
\operatorname{det}\left(\begin{array}{llll}
Q_{11}(\mathbf{n}) & Q_{12}(\mathbf{n}) & Q_{13}(\mathbf{n}) & Q_{14}(\mathbf{n}) \\
Q_{21}(\mathbf{n}) & Q_{22}(\mathbf{n}) & Q_{23}(\mathbf{n}) & Q_{24}(\mathbf{n}) \\
Q_{31}(\mathbf{n}) & Q_{32}(\mathbf{n}) & Q_{33}(\mathbf{n}) & Q_{34}(\mathbf{n}) \\
Q_{41}(\mathbf{n}) & Q_{42}(\mathbf{n}) & Q_{43}(\mathbf{n}) & Q_{44}(\mathbf{n})
\end{array}\right)=0,
$$


where

$$
\begin{aligned}
& Q_{r s}(\mathbf{n})=C_{m s r n} n_{n} n_{m}-\varrho c^{2} \delta_{r s}, \\
& Q_{r 4}(\mathbf{n})=Q_{4 r}(\mathbf{n})=B_{m r} n_{m} \text { for } r, s=1,2,3, \\
& Q_{44}(\mathbf{n})=\xi+A_{r s} n_{r} n_{s}-\varrho \varkappa c^{2} .
\end{aligned}
$$

It is easy to see that all solutions of the equation (4.5) are real numbers. In fact, from (4.4) we have

$$
\begin{aligned}
\varrho c^{2}\left(U_{1}^{2}+U_{2}^{2}+U_{3}^{2}+\varkappa \Phi^{2}\right) & =C_{m n r s} U_{r} U_{n} n_{s} n_{m}+ \\
& +\left(\xi+A_{r s} n_{r} n_{s}\right) \Phi^{2}+2 B_{r s} U_{r} n_{s} \Phi .
\end{aligned}
$$

In view of the strong ellipticity condition (3.6), we can see that the right hand side of (4.7) is a positive definite term and the corresponding roots $c^{2}$ are positive. Thus, all solutions $c_{1}, c_{2}, c_{3}$ and $c_{4}$ of the equation (4.5) are real numbers.

Therefore, for every direction of propagation $\mathbf{n}=\left(n_{1}, n_{2}, n_{3}\right)$ in a centro-symmetric elastic material with voids there exist three orthogonal directions of motion and four progressive waves propagating with the associated speeds of propagation $c_{1}, c_{2}, c_{3}$ and $c_{4}$.

\section{(a) Isotropic poroelastic materials}

For an isotropic poroelastic material the progressive wave (4.2) can propagate with the speeds $c_{1}=c_{2}, c_{3}$ and $c_{4}$, where

$$
\begin{gathered}
c_{1}^{2}=c_{2}^{2}=\frac{\mu}{\varrho}, \\
c_{3}^{2}=\frac{1}{2 \varrho \varkappa}\left\{\xi+\alpha+\varkappa(\lambda+2 \mu)+\sqrt{[\xi+\alpha-\varkappa(\lambda+2 \mu)]^{2}+4 \varkappa \beta^{2}}\right\}, \\
c_{4}^{2}=\frac{1}{2 \varrho \varkappa}\left\{\xi+\alpha+\varkappa(\lambda+2 \mu)-\sqrt{[\xi+\alpha-\varkappa(\lambda+2 \mu)]^{2}+4 \varkappa \beta^{2}}\right\} .
\end{gathered}
$$

Moreover, for $c=c_{1}=c_{2}=\sqrt{\frac{\mu}{\varrho}}$, we have the corresponding linear independent solutions $\left(U_{1}, U_{2}, U_{3}, \Phi\right)^{(1)}$ and $\left(U_{1}, U_{2}, U_{3}, \Phi\right)^{(2)}$ of the algebraic system (4.4)

$$
\begin{aligned}
& \left(U_{1}, U_{2}, U_{3}, \Phi\right)^{(1)}=\left(-n_{3}, 0, n_{1}, 0\right), \\
& \left(U_{1}, U_{2}, U_{3}, \Phi\right)^{(2)}=\left(-n_{2}, n_{1}, 0,0\right),
\end{aligned}
$$

for the direction of propagation $\mathbf{n}=\left(n_{1}, n_{2}, n_{3}\right)$. Thus, the characteristic space for $c^{2}=$ $c_{1}^{2}=c_{2}^{2}$ is generated by the linear independent vectors $\mathbf{U}^{(1)}$ and $\mathbf{U}^{(2)}$. It is easy to see that we have

$$
\mathbf{U}^{(1)} \cdot \mathbf{n}=0, \quad \mathbf{U}^{(2)} \cdot \mathbf{n}=0,
$$

where $\mathbf{U}^{(1)}=\left(-n_{3}, 0, n_{1}\right)$ and $\mathbf{U}^{(2)}=\left(-n_{2}, n_{1}, 0\right)$. Therefore, the progressive waves propagating with speeds $c=c_{1}=c_{2}=\sqrt{\frac{\mu}{\varrho}}$ are transverse waves.

For $c=c_{3}$ we have, up to a scalar factor, the corresponding solution $\left(U_{1}, U_{2}, U_{3}, \Phi\right)^{(3)}$ of the algebraic system (4.4) given by

$$
\left(U_{1}, U_{2}, U_{3}, \Phi^{(3)}\right)=\left(n_{1} \mathfrak{c}, n_{2} \mathfrak{c}, n_{3} \mathfrak{c}, \beta\right),
$$

where

$$
\mathfrak{c}=\varrho \varkappa c_{3}^{2}-\xi-\alpha
$$


while for $c=c_{4}$ we have the corresponding solution $\left(U_{1}, U_{2}, U_{3}, \Phi\right)^{(4)}$ of the algebraic system (4.4) given by

$$
\left(U_{1}, U_{2}, U_{3}, \Phi^{(4)}\right)=\left(n_{1} \mathfrak{a}, n_{2} \mathfrak{a}, n_{3} \mathfrak{a}, \beta\right)
$$

with

$$
\mathfrak{a}=\varrho \varkappa c_{4}^{2}-\xi-\alpha .
$$

Thus, it is easy to see that we have

$$
\mathbf{U}^{(3)} \times \mathbf{n}=\mathbf{0}, \quad \mathbf{U}^{(4)} \times \mathbf{n}=\mathbf{0},
$$

where $\mathbf{U}^{(3)}=\mathfrak{c n}$ and $\mathbf{U}^{(4)}=\mathfrak{a n}$ and hence the progressive waves propagating with speeds $c=c_{3}$ and $c=c_{4}$ are longitudinal waves. The lines spanned by $\mathbf{U}^{(3)}$ and $\mathbf{U}^{(4)}$, respectively, are the characteristic spaces for $c_{3}^{2}$ and $c_{4}^{2}$.

Concluding, we can formulate the following result:

Proposition 4.1. For a strongly elliptic isotropic elastic material with voids the progressive wave defined in (4.2) is either one transverse propagating with the speed $c=c_{1}=c_{2}$ or it is one longitudinal propagating with the speeds $c=c_{3}$ or $c=c_{4}$.

Thus, for a strongly elliptic isotropic elastic materials with voids there are but two types of progressive waves: longitudinal and transverse.

\section{(b) Transverse isotropic poroelastic materials}

In this section we consider the case of strongly elliptic transverse isotropic poroelastic materials. For these types of materials the matrix $\mathbf{Q}$ defined by (4.5) is given by

$$
\begin{aligned}
Q_{11} & =c_{11} n_{1}^{2}+c_{66} n_{2}^{2}+c_{55} n_{3}^{2}-\varrho c^{2}, \\
Q_{22} & =c_{66} n_{1}^{2}+c_{11} n_{2}^{2}+c_{55} n_{3}^{2}-\varrho c^{2}, \\
Q_{33} & =c_{55}\left(n_{1}^{2}+n_{2}^{2}\right)+c_{33} n_{3}^{2}-\varrho c^{2}, \\
Q_{44} & =\xi+a_{11}\left(n_{1}^{2}+n_{2}^{2}\right)+a_{33} n_{3}^{2}-\varrho \varkappa c^{2}, \\
Q_{12} & =Q_{21}=\left(c_{12}+c_{66}\right) n_{1} n_{2}, \\
Q_{13} & =Q_{31}=\left(c_{13}+c_{55}\right) n_{1} n_{3}, \\
Q_{14} & =Q_{41}=b_{11} n_{1}, \\
Q_{23} & =Q_{32}=\left(c_{13}+c_{55}\right) n_{2} n_{3}, \\
Q_{24} & =Q_{42}=b_{11} n_{2}, \\
Q_{34} & =Q_{43}=b_{33} n_{3} .
\end{aligned}
$$

Then the equation (4.5) reduces to

$$
\left[c_{66}\left(n_{1}^{2}+n_{2}^{2}\right)+c_{55} n_{3}^{2}-\varrho c^{2}\right] D=0
$$

where

$$
D=\operatorname{det}\left(q_{i j}\right)
$$

with

$$
\begin{aligned}
& q_{11}=c_{11}\left(n_{1}^{2}+n_{2}^{2}\right)+c_{55} n_{3}^{2}-\varrho c^{2}, \\
& q_{22}=c_{55}\left(n_{1}^{2}+n_{2}^{2}\right)+c_{33} n_{3}^{2}-\varrho c^{2}, \\
& q_{33}=\xi+a_{11}\left(n_{1}^{2}+n_{2}^{2}\right)+a_{33} n_{3}^{2}-\varrho \varkappa c^{2}, \\
& q_{12}=\left(c_{13}+c_{55}\right) n_{3}, \quad q_{21}=\left(c_{13}+c_{55}\right)\left(n_{1}^{2}+n_{2}^{2}\right) n_{3}, \\
& q_{13}=b_{11}, \quad q_{31}=b_{11}\left(n_{1}^{2}+n_{2}^{2}\right), \\
& q_{23}=q_{32}=b_{33} n_{3} .
\end{aligned}
$$


Thus, we can observe that the progressive wave can propagate in an arbitrary direction $\mathbf{n}$ with the first speed

$$
c_{1}^{2}=\frac{1}{\varrho}\left[c_{66}+\left(c_{55}-c_{66}\right) n_{3}^{2}\right] .
$$

The other speeds $c$ are solutions of the equation

$$
c^{6}+\Lambda_{1} c^{4}+\Lambda_{2} c^{2}+\Lambda_{3}=0,
$$

where

$$
\begin{aligned}
\Lambda_{1} & =-\frac{1}{\varrho}\left[\frac{\xi}{\varkappa}+\left(\frac{a_{11}}{\varkappa}+c_{11}+c_{55}\right)+\left(\frac{a_{33}-a_{11}}{\varkappa}+c_{33}-c_{11}\right) n_{3}^{2}\right], \\
\Lambda_{2} & =\frac{1}{\varrho^{2}}\left\{\left[\left(c_{11}+c_{55}\right)+\left(c_{33}-c_{11}\right) n_{3}^{2}\right]\left(\frac{\xi}{\varkappa}+\frac{a_{11}}{\varkappa}+\frac{a_{33}-a_{11}}{\varkappa} n_{3}^{2}\right)+\right. \\
& +\left[c_{11}+\left(c_{55}-c_{11}\right) n_{3}^{2}\right]\left[c_{55}+\left(c_{33}-c_{55}\right) n_{3}^{2}\right]- \\
& \left.-\left(c_{13}+c_{55}\right)^{2}\left(1-n_{3}^{2}\right) n_{3}^{2}-\frac{b_{11}^{2}}{\varkappa}-\frac{b_{33}^{2}-b_{11}^{2}}{\varkappa} n_{3}^{2}\right\}, \\
\Lambda_{3} & =-\frac{1}{\varrho^{3}}\left(\frac{\xi}{\varkappa}+\frac{a_{11}}{\varkappa}+\frac{a_{33}-a_{11}}{\varkappa} n_{3}^{2}\right)\left\{[ c _ { 1 1 } + ( c _ { 5 5 } - c _ { 1 1 } ) n _ { 3 } ^ { 2 } ] \left[c_{55}+\right.\right. \\
& \left.\left.+\left(c_{33}-c_{55}\right) n_{3}^{2}\right]-\left(c_{13}+c_{55}\right)^{2}\left(1-n_{3}^{2}\right) n_{3}^{2}\right\}+\frac{1}{\varrho^{3} \varkappa}\left\{b_{11}^{2}\left[c_{55}+\left(c_{33}-c_{55}\right) n_{3}^{2}\right] .\right. \\
& \left.\cdot\left(1-n_{3}^{2}\right)+b_{33}^{2}\left[c_{11}+\left(c_{55}-c_{11}\right) n_{3}^{2}\right] n_{3}^{2}-2 b_{11} b_{33}\left(c_{13}+c_{55}\right)\left(1-n_{3}^{2}\right) n_{3}^{2}\right\} .
\end{aligned}
$$

In what follows we will discuss the following two directions of propagation: (i) the progressive wave propagates in the plane of isotropy and (ii) the progressive wave propagates along the axis of rotational symmetry $O x_{3}$.

Case (i): $n_{3}=0$

In this case the speeds of propagation are given by

$$
\begin{aligned}
& \tilde{c}_{1}^{2}=\frac{1}{\varrho} c_{66}, \\
& \tilde{c}_{2}^{2}=\frac{1}{\varrho} c_{55}, \\
& \tilde{c}_{3}^{2}=\frac{1}{2 \varrho \varkappa}\left[\xi+a_{11}+\varkappa c_{11}-\sqrt{\left(\xi+a_{11}-\varkappa c_{11}\right)^{2}+4 \varkappa b_{11}^{2}}\right], \\
& \tilde{c}_{4}^{2}=\frac{1}{2 \varrho \varkappa}\left[\xi+a_{11}+\varkappa c_{11}+\sqrt{\left(\xi+a_{11}-\varkappa c_{11}\right)^{2}+4 \varkappa b_{11}^{2}}\right] .
\end{aligned}
$$

The solutions of the system (4.4) corresponding to the speeds $\tilde{c}_{1}, \tilde{c}_{2}, \tilde{c}_{3}$ and $\tilde{c}_{4}$ are, respectively, given by

$$
\begin{gathered}
\left(\tilde{U}_{1}, \tilde{U}_{2}, \tilde{U}_{3}, \tilde{\Phi}\right)^{(1)}=\left(-n_{2}, n_{1}, 0,0\right) \\
\left(\tilde{U}_{1}, \tilde{U}_{2}, \tilde{U}_{3}, \tilde{\Phi}\right)^{(2)}=(0,0,1,0), \\
\left(\tilde{U}_{1}, \tilde{U}_{2}, \tilde{U}_{3}, \tilde{\Phi}\right)^{(3)}=\left(\left(\varrho \varkappa c_{3}^{2}-\xi-a_{11}\right) n_{1},\left(\varrho \varkappa c_{3}^{2}-\xi-a_{11}\right) n_{2}, 0, b_{11}\right), \\
\left(\tilde{U}_{1}, \tilde{U}_{2}, \tilde{U}_{3}, \tilde{\Phi}\right)^{(4)}=\left(\left(\varrho \varkappa c_{4}^{2}-\xi-a_{11}\right) n_{1},\left(\varrho \varkappa c_{4}^{2}-\xi-a_{11}\right) n_{2}, 0, b_{11}\right) .
\end{gathered}
$$

In conclusion, we have the following result: 
Proposition 4.2. For a strongly elliptic transversely isotropic elastic material with voids the progressive waves which propagate in the plane of isotropy are two transverse propagating with the speeds $\tilde{c}_{1}$ or $\tilde{c}_{2}$ and two longitudinal propagating with the speeds $\tilde{c}_{3}$ or $\tilde{c}_{4}$.

Case (ii): $n_{1}=0, n_{2}=0$

If the direction of propagation is along of the axis of rotational symmetry, then the speeds of propagation are

$$
\begin{aligned}
& \hat{c}_{1}^{2}=\hat{c}_{2}^{2}=\frac{1}{\varrho} c_{55}, \\
& \hat{c}_{3}^{2}=\frac{1}{2 \varrho \varkappa}\left[\xi+a_{33}+\varkappa c_{33}-\sqrt{\left(\xi+a_{33}-\varkappa c_{33}\right)^{2}+4 \varkappa b_{33}^{2}}\right], \\
& \hat{c}_{4}^{2}=\frac{1}{2 \varrho \varkappa}\left[\xi+a_{33}+\varkappa c_{33}+\sqrt{\left(\xi+a_{33}-\varkappa c_{33}\right)^{2}+4 \varkappa b_{33}^{2}}\right] .
\end{aligned}
$$

For the double solution of the equation (4.5) the corresponding solutions of the algebraic system (4.4) are given by

$$
\begin{aligned}
& \left(\hat{U}_{1}, \hat{U}_{2}, \hat{U}_{3}, \hat{\Phi}\right)^{(1)}=(1,0,0,0), \\
& \left(\hat{U}_{1}, \hat{U}_{2}, \hat{U}_{3}, \hat{\Phi}\right)^{(2)}=(0,1,0,0),
\end{aligned}
$$

while the corresponding solutions for the speeds $\hat{c}_{3}$ and $\hat{c}_{4}$ are

$$
\begin{aligned}
& \left(U_{1}, U_{2}, U_{3}, \Phi\right)^{(3)}=\left(0,0, \varrho \varkappa \hat{c}_{3}^{2}-\xi-a_{33}, b_{33}\right), \\
& \left(U_{1}, U_{2}, U_{3}, \Phi\right)^{(4)}=\left(0,0, \varrho \varkappa \hat{c}_{4}^{2}-\xi-a_{33}, b_{33}\right) .
\end{aligned}
$$

Thus, we can give the following result:

Proposition 4.3. For a strongly elliptic transversely isotropic elastic material with voids the progressive waves which propagate along the axis of isotropy are two transverse propagating with the speed $\hat{c}_{1}=\hat{c}_{2}$ and two longitudinal propagating with the speeds $\hat{c}_{3}$ or $\hat{c}_{4}$.

\section{Thermal effects}

In this section we study the plane progressive waves in thermoelastic isotropic materials with voids. In the absence of the body force, the extrinsic equilibrated body force and the heat source, the equations which describe the behaviour of the thermoelastic material with voids are given by (cf. Ieşan (2004))

$$
\begin{aligned}
\mu \Delta u_{r}+(\lambda+\mu) u_{j, j r}+\beta \varphi_{, r}-b \theta_{, r} & =\varrho \ddot{u}_{r} \\
\alpha \Delta \varphi-\beta u_{j, j}-\xi \varphi+m \theta & =\varrho \varkappa \ddot{\varphi} \\
k \Delta \theta-T_{0} b \dot{u}_{j, j}-T_{0} m \dot{\varphi} & =T_{0} a \dot{\theta}
\end{aligned}
$$

where $\lambda, \mu, \alpha, \beta, \xi, a, b, m$ and $k$ are constitutive coefficients which describe the thermal properties of material and $T_{0}$ is the ambient absolute temperature. We suppose the material to be strongly elliptic. As a consequence of the entropy inequality we have that $k$ is a positive constant. 
By a plane harmonic wave in a thermoelastic material with voids we mean a set of functions $\left(u_{r}, \varphi, \theta\right)$ on $\mathbb{R}^{3} \times(-\infty, \infty)$ of the following form

$$
\begin{aligned}
u_{r}(\mathbf{x}, t) & =\operatorname{Re}\left\{U_{r} \exp \left[\mathrm{i}\left(n_{s} x_{s}-c t\right)\right]\right\} \\
\varphi(\mathbf{x}, t) & =\operatorname{Re}\left\{\Phi \exp \left[\mathrm{i}\left(n_{s} x_{s}-c t\right)\right]\right\} \\
\theta(\mathbf{x}, t) & =\operatorname{Re}\left\{\Theta \exp \left[\mathrm{i}\left(n_{s} x_{s}-c t\right)\right]\right\}
\end{aligned}
$$

where $\mathbf{U}=\left(U_{1}, U_{2}, U_{3}\right)$ denotes the direction of motion, $\Phi, \Theta$ are complex constants, $\mathbf{n}=\left(n_{1}, n_{2}, n_{3}\right)$ is the direction of propagation and $\left(U_{1}, U_{2}, U_{3}, \Phi, \Theta\right) \neq \mathbf{0}$ and $\operatorname{Re}[c]$ is the speed of propagation. If $\operatorname{Im}[c]<0$ then the waves are damped, whereas if $c$ is a real number the waves are undamped.

By a direct substitution of the expressions of the plane progressive thermoelastic wave described by (5.2) into basic equation (5.1) we obtain that such waves are possible in a thermoelastic material with voids if and only if

$$
\begin{array}{r}
{\left[\left(\mu-\varrho c^{2}\right) \delta_{r j}+(\lambda+\mu) n_{r} n_{j}\right] U_{j}-\mathrm{i} \beta n_{r} \Phi+\mathrm{i} b n_{r} \Theta=0,} \\
\mathrm{i} \beta n_{j} U_{j}+\left(\alpha+\xi-\varrho \varkappa c^{2}\right) \Phi-m \Theta=0 \\
T_{0} b c n_{j} U_{j}-\mathrm{i} T_{0} m c \Phi+\left(k-\mathrm{i} T_{0} a c\right) \Theta=0 .
\end{array}
$$

This linear algebraic system admits a non-zero solution $\left(U_{1}, U_{2}, U_{3}, \Phi, \Theta\right)$ if and only if $c$ is solution of the following equation

$$
\left(\varrho c^{2}-\mu\right)^{2}\left|\begin{array}{ccc}
\lambda+2 \mu-\varrho c^{2} & -\mathrm{i} \beta & \mathrm{i} b \\
\mathrm{i} \beta & \xi+\alpha-\varrho \varkappa c^{2} & -m \\
T_{0} b c & -\mathrm{i} T_{0} c m & k-\mathrm{i} T_{0} a c
\end{array}\right|=0
$$

that is

$$
\left(\varrho c^{2}-\mu\right)^{2}\left(\mathrm{i} \Upsilon_{5} c^{5}+\Upsilon_{4} c^{4}+\mathrm{i} \Upsilon_{3} c^{3}+\Upsilon_{2} c^{2}+\mathrm{i} \Upsilon_{1} c+\Upsilon_{0}\right)=0
$$

where

$$
\begin{aligned}
\Upsilon_{5}= & -a T_{0} \varkappa \varrho^{2}, \Upsilon_{4}=k \varkappa \varrho^{2}, \\
\Upsilon_{3}= & T_{0} \varrho\left\{m^{2}+a \xi+b^{2} \varkappa+a[\alpha+\varkappa(\lambda+2 \mu)]\right\}, \\
\Upsilon_{2}= & -k \varrho[\xi+\alpha+\varkappa(\lambda+2 \mu)], \\
\Upsilon_{1}= & -T_{0}\left\{-2 b m \beta+m^{2}(\lambda+2 \mu)+b^{2} \xi+a\left[-\beta^{2}+(\lambda+2 \mu) \xi\right]+\right. \\
& \left.\quad+\alpha\left[b^{2}+a(\lambda+2 \mu)\right]\right\}, \\
\Upsilon_{0}= & k\left[-\beta^{2}+(\lambda+2 \mu)(\xi+\alpha)\right] .
\end{aligned}
$$

Obviously, the above equation has the double root

$$
c=c_{1}=c_{2}=\sqrt{\frac{\mu}{\varrho}} .
$$

For these values of $c$ we have the following solution of system (5.3)

$$
\begin{aligned}
& \left(U_{1}, U_{2}, U_{3}, \Phi, \Theta\right)=\left(-\left(n_{2}+n_{3}\right), n_{1}, n_{1}, 0,0\right) \text { if } n_{1} \neq 0 \text {, } \\
& \left(U_{1}, U_{2}, U_{3}, \Phi, \Theta\right)=\left(n_{2},-\left(n_{1}+n_{3}\right), n_{2}, 0,0\right) \text { if } n_{2} \neq 0 \text {, } \\
& \left(U_{1}, U_{2}, U_{3}, \Phi, \Theta\right)=\left(n_{3}, n_{3},-\left(n_{1}+n_{2}\right), 0,0\right) \text { if } n_{3} \neq 0 \text {. }
\end{aligned}
$$

It is easy to see that these progressive waves are transverse waves. If we take into consideration the case of transverse waves $(\mathbf{U} \cdot \mathbf{n}=0)$ then from the equations $(5.3)$ we find that the speeds defined by (5.7) are the only admissible speeds for this type of waves. 
In the case of longitudinal waves we have $\mathbf{U} \cdot \mathbf{n}=U$, where $U=|\mathbf{U}|$. For the case of longitudinal waves the system (5.3) becomes

$$
\begin{gathered}
\left(\lambda+2 \mu-\varrho c^{2}\right) U-\mathrm{i} \beta \Phi+\mathrm{i} b \Theta=0, \\
\mathrm{i} \beta U+\left(\alpha+\xi-\varrho \varkappa c^{2}\right) \Phi-m \Theta=0, \\
T_{0} b c U-\mathrm{i} T_{0} m c \Phi+\left(k-\mathrm{i} T_{0} a c\right) \Theta=0 .
\end{gathered}
$$

This system has a nontrivial solution if and only if $c$ is solution of the equation

$$
\mathrm{i} \Upsilon_{5} c^{5}+\Upsilon_{4} c^{4}+\mathrm{i} \Upsilon_{3} c^{3}+\Upsilon_{2} c^{2}+\mathrm{i} \Upsilon_{1} c+\Upsilon_{0}=0
$$

If $b \neq 0$ or $m \neq 0$, then the solutions of this equation are not real numbers. In fact, if we suppose that there is a real solution of the above equation then it has to satisfy both equations below

$$
\begin{gathered}
c\left(\Upsilon_{5} c^{4}+\Upsilon_{3} c^{2}+\Upsilon_{1}\right)=0 \quad \text { and } \\
\Upsilon_{4} c^{4}+\Upsilon_{2} c^{2}+\Upsilon_{0}=0 .
\end{gathered}
$$

But the poroelastic material is strongly elliptic and thus we have that $c=0$ cannot be solution of the equation (5.11) 2 . Actually, for $b \neq 0$ or $m \neq 0$, the equations (5.11) don't have any common solution. In consequence, in this case, the equation (5.10) has only solutions in $\mathbb{C} \backslash \mathbb{R}$.

If $b \rightarrow 0$ and $m \rightarrow 0$, then the equations (5.11) have two common solutions. This case will be discussed as a particular case.

In view of the above discussion we can conclude:

Proposition 5.1. For a thermoelastic isotropic material with voids for which $b \neq 0$ or $m \neq 0$, the progressive wave defined in (5.2) is either one undamped transverse wave propagating with the speeds $c=c_{1}=c_{2}$ or there are three damped longitudinal waves propagating with the speeds $c$ which are solutions of the equation (5.10).

In the linear theory of thermoleastic materials with voids there are three coupling coefficients: $\beta, b$ and $m$. The pair $(b, m)$ couples the equations $(5.1)_{1,2}$ describing the poroelastic behaviour of material with the heat equation $(5.1)_{3}$, while the pair $(\beta, b)$ couples the equations $(5.1)_{1}$ which describe purely the elastic behaviour of material with the porosity and heat equations $(5.1)_{2,3}$.

In what follows we use the convergence results established by Chiriţă \& Ciarletta (2008), in order to discuss how the thermoelastic progressive wave behaves as the coupling coefficients $\beta, b$ and $m$ tend to zero. This discussion helps us to compare the results obtained in thermoporoelastic theory with those established in the poroelastic theory and also with the results from the classical elasticity.

(Case I) $: b \rightarrow 0, m \rightarrow 0$ and $\beta \neq 0$

In this case the possible propagating speeds are

$$
\begin{aligned}
c_{1}^{2} & =c_{2}^{2}=\frac{\mu}{\varrho}, \\
c_{3}^{2} & =\frac{1}{2 \varrho \varkappa}\left\{\xi+\alpha+\varkappa(\lambda+2 \mu)+\sqrt{[\xi+\alpha-\varkappa(\lambda+2 \mu)]^{2}+4 \varkappa \beta^{2}}\right\}, \\
c_{4}^{2} & =\frac{1}{2 \varrho \varkappa}\left\{\xi+\alpha+\varkappa(\lambda+2 \mu)-\sqrt{[\xi+\alpha-\varkappa(\lambda+2 \mu)]^{2}+4 \varkappa \beta^{2}}\right\} \\
c_{5} & =-\frac{i k}{a T_{0}} .
\end{aligned}
$$


For the first four speeds we retrieve the poroelastic case discussed in the Subsection 4.1. The thermal wave is missing for these speeds and the poroelastic waves are not affected by the thermal field.

For the fifth speed we can observe that we cannot have a poroelastic wave but we have a damped thermal wave which are not affected by the poroelastic field.

This behaviour is possible because the equations are non-coupled. In the coupled case the thermal field affects the poroelastic waves and the poroelastic field affects the thermal waves.

(Case II): $\beta \rightarrow 0, b \rightarrow 0$ and $m \neq 0$

In this non-coupled case the possible propagating speeds are

$$
\begin{aligned}
& c_{1}^{2}=c_{2}^{2}=\frac{\mu}{\varrho}, \\
& c_{3}^{2}=\frac{\lambda+2 \mu}{\varrho}, \\
& c_{4}=\frac{\mathrm{i}}{3 a T_{0}}\left[-k+\frac{1}{\varkappa \varrho}\left(\frac{\sqrt[3]{2} p}{q}-\frac{q}{\sqrt[3]{2}}\right)\right], \\
& c_{5}=\frac{\mathrm{i}}{3 a T_{0}}\left[-k-\frac{1}{2 \varkappa \varrho}\left(\frac{\sqrt[3]{2}(1+i \sqrt{3}) p}{q}-\frac{(1-i \sqrt{3}) q}{\sqrt[3]{2}}\right)\right], \\
& c_{6}=\frac{\mathrm{i}}{3 a T_{0}}\left[-k-\frac{1}{2 \varkappa \varrho}\left(\frac{\sqrt[3]{2}(1-i \sqrt{3}) p}{q}-\frac{(1+i \sqrt{3}) q}{\sqrt[3]{2}}\right)\right] .
\end{aligned}
$$

where

$$
\begin{aligned}
p= & \varkappa \varrho\left\{-k^{2} \varkappa \varrho+3 a T^{2}\left[m^{2}+a(\xi+\alpha)\right]\right\}, \\
\Delta= & 4\left[-k^{2} \varkappa \varrho+3 a T_{0}^{2}\left(m^{2}+a(\xi+\alpha)\right)\right]^{3}+ \\
& +k^{2} \varkappa \varrho\left[9 a T_{0}^{2}\left(-m^{2}+2 a \xi\right)+2\left(9 a^{2} T_{0}^{2} \alpha+k^{2} \varkappa \varrho\right)\right]^{2}, \\
q= & \left\{\begin{array}{c}
\left\{\left[-9 a m^{2} T_{0}^{2}+2 k^{2} \varkappa \varrho+18 a^{2} T_{0}^{2}(\xi+\alpha)\right]+\varkappa \varrho \sqrt{\Delta}\right\}^{1 / 3} \text { if } \Delta \geq 0 \\
\left\{\left[-9 a m^{2} T_{0}^{2}+2 k^{2} \varkappa \varrho+18 a^{2} T_{0}^{2}(\xi+\alpha)\right]+i \varkappa \varrho \sqrt{-\Delta}\right\}^{1 / 3} \text { if } \Delta<0 .
\end{array}\right.
\end{aligned}
$$

The waves with the propagated speeds $c_{1}^{2}$ are discussed in the general case. Let now consider the progressive wave corresponding to the speeds $c_{3}^{2}$. For this speed, up to a scalar factor, we find

$$
\left(U_{1}, U_{2}, U_{3}, \Phi, \Theta\right)=\left(n_{1}, n_{2}, n_{3}, 0,0\right) .
$$

We can observe that we retrieve the longitudinal progressive wave from classical elasticity. This fact is a consequence of the decoupling of equations which describe the mechanical behaviour by the porosity equation and heat equation.

Regarding the progressive waves which propagate with the speeds $c_{4,5,6}$ we obtain

$$
\left(U_{1}, U_{2}, U_{3}, \Phi^{(4,5,6)}, \Theta\right)=\left(0,0,0, k-\mathrm{i} T_{0} a c_{4,5,6}, T_{0} m c_{4,5,6}\right) .
$$

These are poro-thermal waves which are not affected by the classical mechanical field but are affected by the presence of voids.

(Case III): $\beta \rightarrow 0, b \rightarrow 0$ and $m \rightarrow 0$

This is a fully uncoupled case. In this case the waves are purely mechanical waves with the speeds

$$
\begin{aligned}
c_{1}^{2} & =c_{2}^{2}=\frac{\mu}{\varrho}, \\
c_{3}^{2} & =\frac{\lambda+2 \mu}{\varrho}
\end{aligned}
$$


or purely porous waves with the speed

$$
c_{4}=\frac{\alpha+\xi}{\varrho \varkappa}
$$

or purely thermal waves with the speed

$$
c_{5}=-\frac{\mathrm{i} k}{a T_{0}} .
$$

Moreover, each field (mechanical, porosity, thermal field) don't affect the waves from the other fields.

\section{Concluding remarks}

The main purpose of this paper was to study the strong ellipticity condition in the theory of materials with voids. To this end the strong ellipticity condition was formulated for general anisotropic elastic materials with voids and relevant results were reported concerning explicit necessary and sufficient conditions characterizing the strong ellipticity of the isotropic and transversely isotropic elastic materials with voids. We have to outline that similar results can be obtained for other elastic materials with voids with special symmetries as, for example, those with rhombic symmetry.

Supposing the material to be strongly elliptic we studied the propagation of progressive waves in isotropic and transversely isotropic poroelastic bodies. It is shown that there are only transverse and longitudinal undamped waves and the explicit expressions are presented for the speeds of propagation. In the context of strongly elliptic poroelastic materials we can also give a good description of the surface waves. The analysis of the behaviour of surface waves in poroelastic materials will be treated in a forthcoming paper.

In the last section we studied the propagation of harmonic plane waves in isotropic thermoelastic materials with voids. Then there are two undamped transverse harmonic waves and three damped longitudinal harmonic waves. When the thermal effect is taken into consideration there are three coefficients coupling the equations that describe purely the elastic behaviour of material with the porosity and heat equations. In the fully coupled case we give a characterization result and then we show how the coupling coefficients affect the propagation of harmonic plane waves. This discussion proves that the present results are in concordance with the results from classical elasticity and also with the results established for poroelastic materials.

The research of the authors was supported by the Romanian Ministry of Education and Research, grant ID-401, 15 / 28.09.2007. The authors would like to express their gratitude to the referees for their careful assessment as well as for their fruitful suggestions regarding the initial version of the paper.

\section{References}

Bunsell, A.R. \& Renard, J. 2005 Fundamentals of fibre reinforced composite materials, CRC. Series: Series in Material Science and Engineering 13. CRC Press Taylor \& Francis Group.

Casas, P.S. \& Quintanilla, R. 2005 Exponential decay in one-dimensional porous thermoelasticity. Mech. Res. Comm. 32, 652-658.

Chiriţă, S. \& Ciarletta, M. 2006 Spatial estimates for the constrained anisotropic elastic cylinder. J. Elasticity 85, 189-213.

Chiriţă, S. 2006 On the strong ellipticity condition for transversely isotropic linearly elastic solids. An. St. Univ. Iasi, Matematica f.2 52, 113-118. 
Chiriţă, S., Danescu, A. \& Ciarletta, M. 2007 On the strong ellipticity of the anisotropic linearly elastic materials. J. Elasticity 87, 1-27.

Chiriţă, S. \& Danescu, A. 2008 Strong ellipticity for tetragonal system in linearly elastic solids. Int. J. Solids Struct. 45, 4850-4859.

Chiriţă, S. \& Ciarletta, M. 2008 On the structural stability of thermoelastic model of porous media. Math. Meth. Appl. Sci. 31, 19-34.

Christov, C.I. \& Jordan, P.M. 2005 Heat conduction paradox involving second sound propagation in moving media, Phys. Rev. Lett. 94, 154301-1-154301-4.

Christov, I., Jordan, P.M. \& Christov, C.I. 2006 Nonlinear acoustic propagation in homentropic perfect gases: A numerical study. Phys. Lett. A 353, 273-280.

Ciarletta, M. \& Ieşan, D. 1993 Non-classical elastic solids. New York: Longman Scientific and Technical, Harlow, Essex, UK and John Wiley\&Sons, Inc..

Ciarletta, M. \& Straughan, B. 2006 Poroacoustic acceleration waves. Proc. R. Soc. Lond. A 462, 3493-3499.

Ciarletta, M. \& Straughan, B. 2007a Poroacoustic acceleration waves with second sound. J. Sound and Vibration 306, 725-731.

Ciarletta, M. \& Straughan, B. 2007b Thermo-poroacoustic acceleration waves in elastic materials with voids. J. Math. Anal. Appl. 333, 142-150.

Ciarletta, M., Straughan, B. \& Zampoli, V. 2007 Thermo-poroacoustic acceleration waves in elastic materials with voids without energy dissipation. Int. J. Eng. Sci. 45, 736-743.

Cowin, S.C. \& Nunziato, J.W. 1983 Linear elastic materials with voids. J. Elasticity 13, 125-147.

Dacorogna, B. 2001 Necessary and sufficient conditions for strong ellipticity of isotropic functions in any dimension. Dyn. Syst. Ser. B 1, 257-263.

Ding, H., Chen, W. \& Zhang, L. 2006 Elasticity of transversely isotropic materials, Series: Solid mechanics and its applications 126. New York: Springer.

Dong, X.N. \& Guo, X.E. 2004 The dependence of transversely isotropic elasticity of human femoral cortical bone on porosity. J. Biomechanics 37, 1281-1287.

Fichera, G. 1965 Linear elliptic differential systems and eigenvalue problems. New York: Springer.

Ghiba, I.D. 2008a Semi-inverse solution for Saint-Venant's problem in the theory of porous elastic materials. Eur. J. Mech. A/Solids 27, 1060-1074.

Ghiba, I.D. 2008b Spatial estimates concerning the harmonic vibrations in rectangular plates with voids, Arch. Mech. 60, 263-279.

Goldmann, T., Seiner, H. \& Landa, M. 2005 Experimental determination of elastic coefficients of dry bovine bone. Bull. Appl. Mech. 1, 262-275.

Goodman, M.A. \& Cowin, S.C. 1972 A Continuum theory for granular materials. Arch. Rational Mech. Anal. 44, 249-266.

Gurtin, M.E. 1972 The linear theory of elasticity. In Handbuch der Physik (ed. Truesdell, C.) vol. VIa/2. New York: Springer, pp. 1-292.

Han, D., Dai, H.H. \& Qi, L. Conditions for strong ellipticity of anisotropic elastic materials. J. Elasticity, DOI 10.1007/s10659-009-9205-5.

Humphrey, J.D. 2002 Cardiovascular solid mechanics. New York: Springer.

Ieşan, D. 1986 A theory of thermoelastic materials with voids. Acta Mech. 60, 67-89.

Ieşan, D. 2004 Thermoelastic models of continua. London: Kluwer Academic Publishers.

Jaeger, J.C., Cook, N.G.W. \& Zimmerman, R.W. 2007 Fundamentals of rock mechanics, 4rd edn. Blackwell Publishing.

Jordan, P.M. \& Christov, C.I. 2005 A simple finite difference scheme for modelling the finite-time blow-up of acoustic acceleration waves. J. Sound Vibration 281, 1207-1216.

Jordan, P.M. \& Puri, A. 2005 Growth/decay of transverse acceleration waves in nonlinear elastic media. Phys. Lett. A 341, 427-434.

Magaña, A. \& Quintanilla, R. 2006 On the time decay of solutions in one-dimensional theories of porous materials, Int. J. Solids Struct. 43, 3414-3427.

Magaña, A. \& Quintanilla, R. 2006 On the spatial behaviour of solutions for porous elastic solids with quasi-static microvoids. Math. Comput. Modelling 44, 710-716.

Merodio, J. \& Ogden, R.W. 2003 Instabilities and loss of ellipticity in fiber-reinforced compressible nonlinearly elastic solids under plane deformation. Int. J. Solids Struct. 40, 4707-4727. 
Merodio, J. \& Ogden, R.W. 2003 A note on strong ellipticity for transversely isotropic linearly elastic solids. Q. J. Mech. Appl. Math. 56 589-591.

Nunziato, J.W. \& Walsh, E.K. 1977 On the influence of void compaction and material nonuniformity on the propagation of one-dimensional acceleration waves in granular materials. Arch. Ration. Mech. Anal. 64, 299-316.

Nunziato, J.W. \& Walsh, E.K. 1978 Addendum "On the influence of void compaction and material non-uniformity on the propagation of one-dimensional acceleration waves in granular materials". Arch. Ration. Mech. Anal. 67, 395-398.

Nunziato, J.W., Kennedy, J.E. \& Walsh, E.K. 1978 The behavior of one-dimensional acceleration waves in an inhomogeneous granular solid. Int. J. Eng. Sci. 16, 637-648.

Nunziato, J.W. \& Cowin, S.C. 1979 A nonlinear theory of elastic materials with voids. Arch. Ration. Mech. Anal. 72, 175-201.

Ostoja-Starzewski, M. \& Trebicki, J. 1999 On the growth and decay of acceleration waves in random media. Proc. R. Soc. Lond. Ser. A 455, 2577-2614.

Padovani, C. 2002 Strong ellipticity of transversely isotropic elasticity tensors. Meccanica 37, $515-525$.

Payton, R.G. 1983 Elastic Wave Propagation in Transversely Isotropic Media. Martinus Nijhoff, The Hague.

Puri, P. \& Jordan, P.M. 2004 On the propagation of plane waves in type-III thermoelastic media. Proc. R. Soc. Lond. Ser. A 460, 3203-3221.

Quintanilla, R. 2001 On uniqueness and continuous dependence in the nonlinear theory of mixtures of elastic solids with voids. Math. Mech. Solids 6, 281-298.

Quintanilla, R. \& Straughan, B. 2004 Discontinuity waves in type III thermoelasticity. Proc. R. Soc. Lond. Ser. A 460, 1169-1175.

Simpson, H.C. \& Spector, S.J. 1983 On copositive matrices and strong ellipticity for isotropic elastic materials. Arch. Ration. Mech. Anal. 84, 55-68.

Spencer, A.J.M. 1992 Continuum theory of the mechanics of fiber-reinforced composites. New York: Springer.

Straughan, B. 2008 Stability and wave motion in porous media. New York: Springer.

Wyllie, D.C. 1999 Foundations on rock: second edition. Taylor \& Francis. 